MATHEMATICS OF COMPUTATION

Volume 68, Number 228, Pages 1615-1621

S 0025-5718(99)01128-X

Article electronically published on March 4, 1999

\title{
TAU METHOD APPROXIMATION OF A GENERALIZED EPSTEIN-HUBBELL ELLIPTIC-TYPE INTEGRAL
}

\author{
H. G. KHAJAH
}

\begin{abstract}
We consider the evaluation of a recent generalization of the Epstein-Hubbell elliptic-type integral using the tau method approximation with a Chebyshev polynomial basis. This also leads to an approximation of Lauricella's hypergeometric function of three variables. Numerical results are given for polynomial approximations of degree 6 .
\end{abstract}

\section{INTRODUCTION}

The Epstein-Hubbell elliptic-type integral

$$
\Omega_{j}(k)=\int_{0}^{\pi}\left(1-k^{2} \cos \vartheta\right)^{-j-1 / 2} d \vartheta, \quad 0 \leq k<1, j=0,1,2, \ldots,
$$

occurs in connection with certain problems related to the computation of the radiation field off-axis from a uniform circular disk radiating according to an arbitrary angular distribution law $[2,5]$. It has undergone a number of generalizations [1], the most recent of which was introduced by Kalla and Tuan [8] in the form

$$
\begin{aligned}
& \Lambda_{(\lambda, \gamma, \mu)}^{(\alpha, \beta)}(\rho, \delta ; k) \\
& \quad=\int_{0}^{\pi} \frac{\cos ^{2 \alpha-1}(\vartheta / 2) \sin ^{2 \beta-1}(\vartheta / 2)}{\left(1-k^{2} \cos \vartheta\right)^{\mu+1 / 2}}\left[1-\rho \sin ^{2}(\vartheta / 2)\right]^{-\lambda}\left[1+\delta \cos ^{2}(\vartheta / 2)\right]^{-\gamma} d \vartheta,
\end{aligned}
$$

where $0 \leq k<1$. In this paper, the tau method is used to obtain polynomial approximations to the above integral when the parameters and exponents are real numbers ${ }^{1}$ such that $\alpha, \beta>0 ;|\delta|,|\rho|<1$.

Introducing a new variable $\omega=\sin ^{2}(\vartheta / 2)$ transforms the above integral into the form

$(1)$

$$
\Lambda_{(\lambda, \gamma, \mu)}^{(\alpha, \beta)}(\rho, \delta ; k)=(1+\delta)^{-\gamma}\left(1-k^{2}\right)^{-\mu-1 / 2} \int_{0}^{1} \omega^{\beta-1}(1-\omega)^{\alpha-1} v(\omega) d \omega
$$

where $v(\omega)$ is given by

$$
v(\omega)=(1-\rho \omega)^{-\lambda}\left(1-\delta_{0} \omega\right)^{-\gamma}\left(1-k_{0} \omega\right)^{-\mu-1 / 2}
$$

Received by the editor May 16, 1998.

1991 Mathematics Subject Classification. Primary 33C65, 41A10; Secondary 65D20.

Key words and phrases. Tau method approximation, elliptic-type integrals, hypergeometric functions.

${ }^{1}$ With the exception of $k$, they are assumed to be complex numbers in [8]. 
and $\delta_{0}=\delta /(1+\delta), k_{0}=2 k^{2} /\left(k^{2}-1\right)$ for $0 \leq k<1$. The function $v(\omega)$ solves the linear differential equation

$$
\mathcal{D} v:=P_{0}(\omega) v^{\prime}-P_{1}(\omega) v=0, \quad v(0)=1,
$$

with variable coefficients

$$
P_{0}(\omega)=1-A_{0} \omega+B_{0} \omega^{2}-C_{0} \omega^{3}, \quad P_{1}(\omega)=A_{1}-B_{1} \omega+C_{1} \omega^{2},
$$

in which $A_{0}=\rho+\delta_{0}+k_{0}, B_{0}=\rho \delta_{0}+\rho k_{0}+\delta_{0} k_{0}, C_{0}=\rho \delta_{0} k_{0}$ and

$$
\begin{aligned}
& A_{1}=\lambda \rho+\gamma \delta_{0}+\left(\mu+\frac{1}{2}\right) k_{0}, \\
& B_{1}=(\lambda+\gamma) \rho \delta_{0}+\left(\lambda+\mu+\frac{1}{2}\right) \rho k_{0}+\left(\gamma+\mu+\frac{1}{2}\right) \delta_{0} k_{0}, \\
& C_{1}=\left(\lambda+\gamma+\mu+\frac{1}{2}\right) C_{0},
\end{aligned}
$$

The integral (1) is also expressed in terms of Lauricella's hypergeometric function of three variables $F_{D}^{(3)}$ (see [3] and [8]):

$$
\begin{aligned}
& \Lambda_{(\lambda, \gamma, \mu)}^{(\alpha, \beta)}(\rho, \delta ; k) \\
& \quad=B(\alpha, \beta)(1+\delta)^{-\gamma}\left(1-k^{2}\right)^{-\mu-1 / 2} F_{D}^{(3)}\left(\beta, \lambda, \gamma, \mu+\frac{1}{2} ; \alpha+\beta ; \rho, \delta_{0}, k_{0}\right)
\end{aligned}
$$

where $B(\alpha, \beta)$ denotes the beta function. The asymptotic behavior of $\Lambda$ as $k$ tends to 1 is treated in [8], where it is separated into two cases depending on whether or not $\mu-\beta+1 / 2$ is an integer. The required approximation of the integral $\Lambda$ in (1) is obtained from the tau method approximant of $v(\omega)$ in conjunction with the identity

$$
\int_{0}^{1} x^{r-1}(1-x)^{s-1} d x=\frac{\Gamma(r) \Gamma(s)}{\Gamma(r+s)}=B(r, s)
$$

\section{TAU METHOD APPROXIMATION}

This method is based on the idea of an 'economized approximation' due to Lanczos $[9,10]$. In what follows, the recursive formulation of the tau method is applied (see $[11,12])$. The linear operator $\mathcal{D}$ acting on $\omega^{n}(n \geq 0)$ yields

$$
\mathcal{D} \omega^{n}=n \omega^{n-1}-\left(n A_{0}+A_{1}\right) \omega^{n}+\left(n B_{0}+B_{1}\right) \omega^{n+1}-\left(n C_{0}+C_{1}\right) \omega^{n+2} .
$$

If $Q_{i}(\omega)$ is defined in such a way that $\mathcal{D} Q_{i}=\omega^{i}$, then by the linearity of $\mathcal{D}$ the above equation becomes

$$
\mathcal{D} \omega^{n}=\mathcal{D}\left[n Q_{n-1}-\left(n A_{0}+A_{1}\right) Q_{n}+\left(n B_{0}+B_{1}\right) Q_{n+1}-\left(n C_{0}+C_{1}\right) Q_{n+2}\right],
$$

and this in turn leads to the recurrence relation

$$
n Q_{n-1}-\left(n A_{0}+A_{1}\right) Q_{n}+\left(n B_{0}+B_{1}\right) Q_{n+1}-\left(n C_{0}+C_{1}\right) Q_{n+2}=\omega^{n},
$$


which generates the canonical polynomials $Q_{n}$. Three special cases are to be considered at this point:

1. If $C_{0} \neq 0$, then $\left\{Q_{0}, Q_{1}, 1, \omega, \ldots, \omega^{n-2}\right\}$ is the generating set for $Q_{n}, n \geq 2$.

2. If $C_{0}=0$ and $n B_{0}+B_{1} \neq 0$ for all $n \geq 0$, then $Q_{n}$ is generated by the set $\left\{Q_{0}, 1, \omega, \ldots, \omega^{n-1}\right\}$ for $n \geq 1$.

3. If $C_{0}=B_{0}=B_{1}=0$, then $C_{1}=0$ and the generating set is $\left\{1, \omega, \ldots, \omega^{n}\right\}$, $n \geq 0$.

Let $T_{n}^{*}(\omega)$ denote the $n$-th Chebyshev polynomial defined on the unit interval with coefficients $c_{j}^{(n)}(j=0,1, \ldots, n)$ given by

$$
c_{j}^{(n)}=(-1)^{n+j} 2^{2 j-1}\left[2\left(\begin{array}{c}
n+j \\
n-j
\end{array}\right)-\left(\begin{array}{c}
n+j-1 \\
n-j
\end{array}\right)\right] .
$$

For a fixed positive integer $N$, we define the polynomial

$$
V(\omega)=\sum_{i=0}^{\nu} \tau_{i} \sum_{j=0}^{N+\nu-i} c_{j}^{(N+\nu-i)} Q_{j}(\omega)
$$

where $\nu=2,1$ or 0 according to the special cases $1-3$, respectively. Hence $V$ is an exact solution to the perturbed equation

$$
\mathcal{D} V=\sum_{i=0}^{\nu} \tau_{i} T_{N+\nu-i}^{*}
$$

derived from (3) with the same initial condition $V(0)=1$. The unknown $\tau$ parameters are determined from the initial condition on $V$ and, if applicable, by setting the coefficients of $Q_{0}$ and/or $Q_{1}$ in (7) equal to zero. The resulting accuracy of $V$ depends on how small these $\tau$ parameters are, which in turn depends on the prescribed degree $N$. After some algebraic manipulations on (7), $V$ is expressed in the form

$$
V(\omega)=\sum_{n=0}^{N} a_{n} \omega^{n}
$$

in which $a_{0}=1$ while the other coefficients are given in terms of the constant parameters $\alpha, \beta, \lambda, \ldots$. We now replace $v$ in (1) with $V$ and use (5) to get

$$
\int_{0}^{1} \omega^{\beta-1}(1-\omega)^{\alpha-1} V(\omega) d \omega=\sum_{n=0}^{N} B(\alpha, \beta+n) a_{n}=B(\alpha, \beta) \sum_{n=0}^{N} \frac{(\beta)_{n}}{(\alpha+\beta)_{n}} a_{n},
$$

where $(\cdot)_{n}$ is the Pochhammer symbol. Then the desired approximation of (1) becomes

$$
\Lambda_{(\lambda, \gamma, \mu)}^{(\alpha, \beta)}(\rho, \delta ; k) \simeq B(\alpha, \beta)(1+\delta)^{-\gamma}\left(1-k^{2}\right)^{-\mu-1 / 2} \sum_{n=0}^{N} \frac{(\beta)_{n}}{(\alpha+\beta)_{n}} a_{n},
$$

and a comparison of this equation with (4) leads to the following approximation of Lauricella's function:

$$
F_{D}^{(3)}\left(\beta, \lambda, \gamma, \mu+\frac{1}{2} ; \alpha+\beta ; \rho, \delta_{0}, k_{0}\right) \simeq \sum_{n=0}^{N} \frac{(\beta)_{n}}{(\alpha+\beta)_{n}} a_{n}
$$

These approximations are valid for values of $k<1$, and we need to resort to the asymptotic formulas for $\Lambda$ as $k \rightarrow 1$ (see [8]). 


\section{NumERICAL RESUltS}

We have used Mathematica [13] for the algebraic manipulations and to obtain numerical results. However, because of the extreme complexity of the algebraic expressions involved we were unable to present the relevant formulas in closed form. Therefore, it is more convenient to define the desired values for the parameters $\alpha, \beta, \lambda, \ldots$, etc., before computing the coefficients in the polynomial $V(\omega)$ and, consequently, the approximate values of $\Lambda$ in (11). To test the validity of the tau method, the special case where $\delta=\rho=0$ and $\beta=\gamma-\alpha$ is considered (case 3 above); this leads to

$$
\Lambda_{(\lambda, \gamma, \mu)}^{(\alpha, \gamma-\alpha)}(0,0 ; k)=R_{\mu}(k, \alpha, \gamma)
$$

in which $\lambda$ plays an idle role. The elliptic-type integrals $R$ were developed in $[4,6,7]$. For the case at hand, the canonical polynomials are given by

$$
Q_{n}=-\sum_{i=0}^{n} \frac{n !}{i !}\left[\prod_{j=i}^{n} \frac{1}{k_{0}(j+\mu+1 / 2)}\right] \omega^{i}, \quad n \geq 0
$$

and, according to (7), $V(\omega)=\tau_{0} \sum c_{n}^{(N)} Q_{n}(\omega)$ with

$$
\tau_{0}=-\left[\sum_{n=0}^{N} c_{n}^{(N)} \frac{n !}{(\mu+1 / 2)_{n+1}}\left(\frac{k^{2}-1}{2 k^{2}}\right)^{n+1}\right]^{-1} .
$$

Two factors, namely $k$ and $\mu$, determine the magnitude of $\tau_{0}$, and hence the error $v-V$. As $k$ approaches 1 , the terms in the summation above come very close to zero, which leads to a large value for $\tau_{0}$ regardless of the choice of $N$. On the other hand, for a fixed $k<1$, large values of $\mu$ also lead to large $\tau_{0}$ but, nevertheless, this difficulty may be overcome by a proper choice of $N$, or by using the recursion relations on $\mu[4]$, namely

$$
\begin{aligned}
R_{\mu}(k, \alpha, \gamma)= & \frac{\gamma-\mu+1 / 2}{(\mu-1 / 2)\left(1-k^{4}\right)} R_{\mu-2}(k, \alpha, \gamma) \\
& +\frac{(2 \mu-\gamma-1)\left(1+k^{2}\right)-(2 \mu-2 \gamma+2 \alpha-1) k^{2}}{(\mu-1 / 2)\left(1-k^{4}\right)} R_{\mu-1}(k, \alpha, \gamma)
\end{aligned}
$$

For $k \leq 0.5$ and a polynomial approximation of degree $N=6$ our results are found to be in agreement with those tabulated in [6].

With the same degree of approximation $N=6$, numerical values for the general case of $\Lambda$ according to (11) are computed for different values of the parameters (Table 1). Moreover, keeping all the other parameters fixed, the behavior of $\Lambda$ as $k$ approaches 1 is noted for the cases where $\mu-\beta+1 / 2$ is not an integer (Table 2), a positive integer (Table 3 ) and a negative integer (Table 4 ); the errors at $k=0.95$ are found to be, respectively, $1,0.85$ and 0.0014 . This is also evident from the increasing values of $|\tau|$ as $k$ approaches 1 . 
TABLE 1. Values of $\Lambda$ as in (11) for different values of the parameters

\begin{tabular}{|c|c|c|c|c|c|}
\hline$\alpha$ & 0.50 & 0.50 & 1.00 & 0.50 & 2.00 \\
$\beta$ & 0.50 & 1.00 & 0.50 & 2.00 & 0.50 \\
$\gamma$ & 0.25 & -0.75 & -1.00 & 1.50 & -0.50 \\
$\delta$ & 0.33 & -0.50 & -0.50 & 0.90 & -0.33 \\
$k$ & 0.01 & 0.20 & 0.30 & 0.50 & 0.10 \\
$\lambda$ & -2.00 & 1.00 & 1.33 & -0.50 & 0.50 \\
$\mu$ & -3.50 & 1.50 & 2.00 & -1.00 & 1.00 \\
$\rho$ & -0.25 & 0.66 & 0.66 & -0.82 & 0.25 \\
\hline$\left|\tau_{0}\right|$ & $9.0 \times 10^{-14}$ & $6.3 \times 10^{-5}$ & $3.5 \times 10^{-4}$ & $4.3 \times 10^{-5}$ & $7.6 \times 10^{-11}$ \\
$\left|\tau_{1}\right|$ & $6.0 \times 10^{-9}$ & $2.8 \times 10^{-3}$ & $7.8 \times 10^{-3}$ & $6.8 \times 10^{-4}$ & $1.4 \times 10^{-8}$ \\
$\left|\tau_{2}\right|$ & $1.6 \times 10^{-7}$ & $1.6 \times 10^{-2}$ & $3.7 \times 10^{-2}$ & $2.7 \times 10^{-3}$ & $3.1 \times 10^{-7}$ \\
\hline$\Lambda$ & 3.871035 & 3.513762 & 2.355496 & 1.525855 & 1.183366 \\
\hline \hline$\alpha$ & 1.00 & 1.50 & 1.50 & 1.00 & 2.00 \\
$\beta$ & 1.00 & 1.00 & 1.50 & 1.50 & 2.00 \\
$\gamma$ & 0.50 & -0.25 & 0.75 & 2.00 & 1.00 \\
$\delta$ & 0.33 & -0.33 & 0.50 & 0.90 & 0.50 \\
$k$ & 0.10 & 0.01 & 0.20 & 0.66 & 0.30 \\
$\lambda$ & -1.66 & 0.10 & -1.33 & -0.10 & -1.10 \\
$\mu$ & -3.00 & 0.25 & -2.00 & -0.25 & -1.50 \\
$\rho$ & -0.25 & 0.25 & -0.66 & -0.82 & -0.66 \\
\hline$\left|\tau_{0}\right|$ & $5.0 \times 10^{-11}$ & $4.0 \times 10^{-13}$ & $3.7 \times 10^{-8}$ & $1.2 \times 10^{-4}$ & $1.9 \times 10^{-7}$ \\
$\left|\tau_{1}\right|$ & $2.3 \times 10^{-8}$ & $7.3 \times 10^{-9}$ & $3.0 \times 10^{-6}$ & $1.3 \times 10^{-3}$ & $7.0 \times 10^{-6}$ \\
$\left|\tau_{2}\right|$ & $5.1 \times 10^{-7}$ & $1.3 \times 10^{-7}$ & $2.5 \times 10^{-5}$ & $3.2 \times 10^{-3}$ & $4.8 \times 10^{-5}$ \\
\hline$\Lambda$ & 1.139618 & 0.636796 & 0.502315 & 0.397895 & 0.188224 \\
\hline
\end{tabular}

TABle 2. $\alpha=1.5, \beta=1, \gamma=-0.25, \delta=-0.33, \lambda=0.1, \mu=$ $0.25, \rho=0.25, \mu-\beta+1 / 2=-0.25$ non-integer

\begin{tabular}{|c|l|c|l|c|}
\hline$k$ & \multicolumn{1}{|c|}{$\left|\tau_{0}\right|$} & $\left|\tau_{1}\right|$ & $\left|\tau_{2}\right|$ & $\Lambda$ \\
\hline 0.10 & $4.4 \times 10^{-11}$ & $7.0 \times 10^{-9}$ & $1.4 \times 10^{-7}$ & 0.6376688 \\
0.30 & $7.7 \times 10^{-10}$ & $3.3 \times 10^{-9}$ & $2.3 \times 10^{-7}$ & 0.6456921 \\
0.50 & $8.0 \times 10^{-8}$ & $2.8 \times 10^{-7}$ & $1.1 \times 10^{-5}$ & 0.6675759 \\
0.70 & $2.0 \times 10^{-5}$ & $6.9 \times 10^{-5}$ & $2.8 \times 10^{-3}$ & 0.7209109 \\
0.90 & $3.6 \times 10^{-3}$ & $1.2 \times 10^{-2}$ & $5.1 \times 10^{-1}$ & 0.8777903 \\
0.95 & $2.3 \times 10^{-2}$ & $7.9 \times 10^{-2}$ & 3.2 & 0.9503844 \\
\hline
\end{tabular}


TABLE 3. $\alpha=2, \beta=0.5, \gamma=-0.5, \delta=-0.33, \lambda=0.5, \mu=1, \rho=$ $0.25, \mu-\beta+1 / 2=1$ positive integer

\begin{tabular}{|c|l|l|l|c|}
\hline$k$ & \multicolumn{1}{|c|}{$\left|\tau_{0}\right|$} & \multicolumn{1}{|c|}{$\left|\tau_{1}\right|$} & \multicolumn{1}{|c|}{$\left|\tau_{2}\right|$} & $\Lambda$ \\
\hline 0.10 & $7.6 \times 10^{-11}$ & $1.4 \times 10^{-8}$ & $3.1 \times 10^{-7}$ & 1.183366 \\
0.30 & $9.0 \times 10^{-10}$ & $9.7 \times 10^{-9}$ & $4.3 \times 10^{-7}$ & 1.273127 \\
0.50 & $3.3 \times 10^{-7}$ & $8.5 \times 10^{-7}$ & $4.5 \times 10^{-5}$ & 1.516813 \\
0.70 & $8.2 \times 10^{-5}$ & $2.0 \times 10^{-4}$ & $1.1 \times 10^{-2}$ & 2.191343 \\
0.90 & $1.2 \times 10^{-2}$ & $2.8 \times 10^{-2}$ & 1.6 & 5.774337 \\
0.95 & $6.4 \times 10^{-2}$ & $1.6 \times 10^{-1}$ & 8.8 & 10.697969 \\
\hline
\end{tabular}

TABLE 4. $\alpha=1.5, \beta=1.5, \gamma=0.75, \delta=0.5, \lambda=-1.33, \mu=$ $-2, \rho=-0.66, \mu-\beta+1 / 2=-3$ negative integer

\begin{tabular}{|c|c|c|c|c|}
\hline$k$ & $\left|\tau_{0}\right|$ & $\left|\tau_{1}\right|$ & $\left|\tau_{2}\right|$ & $\Lambda$ \\
\hline 0.10 & $7.0 \times 10^{-9}$ & $2.0 \times 10^{-6}$ & $1.9 \times 10^{-5}$ & 0.4995507 \\
0.30 & $1.3 \times 10^{-7}$ & $5.3 \times 10^{-6}$ & $3.9 \times 10^{-5}$ & 0.5071118 \\
0.50 & $1.2 \times 10^{-6}$ & $2.5 \times 10^{-5}$ & $1.2 \times 10^{-4}$ & 0.5240502 \\
0.70 & $1.2 \times 10^{-5}$ & $1.9 \times 10^{-4}$ & $5.8 \times 10^{-4}$ & 0.5539706 \\
0.90 & $7.9 \times 10^{-4}$ & $6.8 \times 10^{-3}$ & $2.6 \times 10^{-2}$ & 0.6021379 \\
0.95 & $9.0 \times 10^{-3}$ & $5.6 \times 10^{-2}$ & $5.4 \times 10^{-1}$ & 0.6170454 \\
\hline
\end{tabular}

\section{ACKNOWLEDGMENT}

The author wishes to thank Professor Shyam Kalla for introducing him to this problem.

\section{REFERENCES}

[1] A. Al-Zamel and S. Kalla. Epstein-Hubbell elliptic-type integral and its generalizations. Appl. Math. Comp., 77:9-32, 1996. MR 97c:33017

[2] L.F. Epstein and J.H. Hubbell. Evaluation of a generalized elliptic-type integral. J. Res. NBS, 67B:1-17, 1963. MR 28:4152

[3] H. Exton. Multiple Hypergeometric Functions and Applications. Ellis Horwood Ltd., New York, 1976. MR 54:10699

[4] M.L. Glasser and S.L. Kalla. Recursion relations for a class of generalized elliptic-type integrals. Rev. Tec. Ing. Univ. Zulia, 12:47-50, 1989. MR 91b:33024

[5] J.H. Hubbell, R.L. Bach, and R.J. Herbold. Radiation field from a circular disk source. J. Res. NBS, 65C:249-264, 1961.

[6] S.L. Kalla, S. Conde, and J.H. Hubbell. Some results on generalized elliptic-type integrals. Appl. Anal., 22:273-287, 1986. MR 88a:33002

[7] S.L. Kalla, C. Leubner, and J.H. Hubbell. Further results on generalized elliptic-type integrals. Appl. Anal., 25:269-274, 1987. MR 88i:33002

[8] S.L. Kalla and V.K. Tuan. Asymptotic formulas for generalized elliptic-type integrals. In Proc. of the Intl. Wkshp. on Recent Advances in Applied Maths., Kuwait, 1996. Kuwait Univ. KFAS.

[9] C. Lanczos. Trigonometric interpolation of empirical and analytical functions. J. Math. Phys., 17:123-199, 1938.

[10] C. Lanczos. Applied Analysis. Prentice-Hall, New Jersey, 1956. MR 15:823c

[11] E.L. Ortiz. The Tau Method. SIAM J. Numer. Anal., 6:480-492, 1969. MR 41:2934 
[12] E.L. Ortiz. Canonical polynomials in the Lanczos' Tau Method. In B.K.P. Scaife, editor, Studies in Numerical Analysis, pages 73-93. Academic Press, New York, 1974. MR 57:14478

[13] Wolfram Research, Inc. Mathematica. Wolfram Research, Inc., Champaign, Illinois, 1994. Version 2.2 .

Applied Sciences Department, College of Technical Studies - Paaet, P.O. Box 66814, BAYAN 43759, KUWAIT

E-mail address: hkhajah@kuc01.kuniv.edu.kw 